\title{
EFECTOS DE UN PROGRAMA DE ENTRENAMIENTO CONCURRENTE SOBRE EL PERFIL ANTROPOMÉTRICO Y LA FUERZA MUSCULAR EN UN GRUPO DE JÓVENES UNIVERSITARIOS
}

\author{
EFFECTS OF A CONCURRENT TRAINING PROGRAM ON ANTHROMETRIC PROFILE \\ AND MUSCULAR STRENGTH IN A GROUP OF UNIVERSITY STUDENTS
}

Cómo citar: Pirazán, J., Rivera, M., Anzola, F., \& Osuna, J. (2019). Efectos de un programa de entrenamiento concurrente sobre el perfil antropométrico y la fuerza muscular en un grupo de jóvenes universitarios. R. Actividad fis. y deporte. 6 (1): 14-31.

Artículo de acceso abierto publicado por: Revista Digital: Actividad Física y Deporte, bajo una licencia Creative Commons CC BY-NC 4.0.

\section{Milton Javier Pirazán Rodríguez}

Licenciado en Educación Física Recreación y Deportes, Maestrante en Pedagogía de la Cultura Física, Universidad Pedagógica y Tecnológica de Colombia. Docente tiempo completo Universidad Santo Tomás Seccional Tunja, Boyacá.

E-mail: milton.pirazan@usantoto.edu.co

\section{Miguel Esteban Rivera Santisteban}

Profesional en Cultura Física Deporte y Recreación, Maestrante en Pedagogía de la Cultura Física, Universidad Pedagógica y Tecnológica de Colombia, Formador Deportivo Instituto Distrital de Recreación y Deporte (IDRD) Bogotá.

E-mail: miguelcoach10@gmail.com

\section{Fernando Anzola Martínez}

Profesional en Cultura Física Deporte y Recreación, Maestrante en Pedagogía de la Cultura Física, Universidad Pedagógica y Tecnológica de Colombia, Docente Educación Física, Bogotá, D.C. E-mail: fergavi13@hotmail.com

\section{Jean Pierr Osuna Fautoque}

Profesional en Cultura Física Deporte y Recreación, Maestrante en Pedagogía de la Cultura Física, Universidad Pedagógica y Tecnológica de Colombia. Docente Educación Física.

E-mail: janpi04@hotmail.com edufisico2019@outlook.com

\section{RESUMEN}

Introducción: Existen cifras alarmantes con relación al sobrepeso y la obesidad, originadas en el desequilibrio energético entre las calorías consumidas y gastadas. Además, de un descenso de la actividad física. Objetivo: Determinar los efectos de un programa de Entrenamiento Concurrente (EC) sobre el perfil antropométrico y la Fuerza Muscular (FM) en jóvenes universitarios con sobrepeso $u$ obesidad. Población y métodos: La muestra está compuesta por 12 
jóvenes entre 18 a 22 años de edad. Se valoró el índice de Masa Corporal (IMC), el porcentaje Masa Grasa (\%MG) y la FM, antes y después de la intervención. Los sujetos completaron un programa de entrenamiento concurrente 3 veces por semana, con sesiones de 60 minutos por día, durante 10 semanas. Resultados: Después de la intervención se observó una reducción significativa en el \%MG ( $p<0,000)$. Sí, bien hubo una reducción en el IMC, no fue estadísticamente significativa ( $p<0,087$ ).

Además, la FM del tren inferior aumentó $39,5 \%$ y del tren superior $24,9 \%$ evaluadas en una Repetición Máxima (1 RM) con un valor de significancia ( $p<0,05)$. Conclusión: Los resultados sugieren que 10 semanas de entrenamiento concurrente, tres veces por semana y 60 minutos por sesión con ejercicios de fuerza y ejercicio aeróbico consiguieron reducir el \%MG, con una reducción en el IMC que no fue estadísticamente significativa, mejorando el perfil antropométrico y se notó en paralelo de forma general, un aumento en la FM.

Palabras Clave: Entrenamiento concurrente; IMC; masa grasa; fuerza muscular; jóvenes con sobrepeso.

\section{ABSTRACT}

Introduction: There are alarming figures in relation to overweight and obesity, originating in the energy imbalance between calories consumed and spent, as well as a decrease in physical activity. Objective: To determine the effects of a Concurrent Training (EC) program on the anthropometric profile and Muscle Strength (FM) in university students who are overweight or obese. Population and methods: The sample is composed of 12 young people between 18 and 22 years of age. The Body Mass Index (BMI), the percentage of Fatty Mass (\% MG) and $\mathrm{FM}$, before and after the intervention were assessed. The subjects complete a concurrent training program 3 times a week, with sessions of 60 minutes per day, for 10 weeks. Results: After the intervention, a significant reduction in\% MG was detected ( $p$ $<0.000$ ). Although there was a reduction in $B M I$, it was not statistically significant ( $p$ $<0.087$ ). In addition, the FM of the lower train increases $39.5 \%$ and the higher train $24.9 \%$ evaluated in a Maximum Repetition (1RM) with a significance value ( $p<0.05)$. Conclusion: The results that affect 10 weeks of concurrent training, three times per week and 60 minutes per session with strength exercises and aerobic exercise were able to reduce the $\% \mathrm{MG}$, with a reduction in $\mathrm{BMI}$ that was not statistically significant, improving the anthropometric profile and a general increase in FM was noted in parallel.

Key Words: Concurrent training; BMI muscle mass fat strength; Overweight young people. 


\section{INTRODUCCIÓN}

La sociedad actual se expone a una vida altamente inactiva, se vuelve cada vez más sedentaria y expuesta a las diferentes Enfermedades No Transmisibles (ENT) entendidas por la Organización Mundial de la Salud (2017) "como enfermedades crónicas, tienden a ser de larga duración y resultan de la combinación de factores genéticos, fisiológicos, ambientales y conductuales" (OMS par. 2). En este sentido, el sobrepeso y la obesidad son enfermedades que se desarrollan descontroladamente. la OMS (2018) estima que "en 2016, más de 1900 millones de adultos de 18 o más años tenían sobrepeso, de los cuales, más de 650 millones eran obesos" (OMS par. 1). y las define como una acumulación anormal o excesiva de grasa que puede ser perjudicial para la salud (OMS, 2017). Estas patologías se desarrollan por diferentes factores como es el desequilibrio energético entre las calorías consumidas y gastadas y una segunda es el descenso de la actividad física por la naturaleza cada vez más sedentaria (OMS, 2018). Igualmente, "Ios entornos alimentarios actuales explotan las vulnerabilidades biológicas, psicológicas, sociales y económicas de las personas, lo que les facilita comer alimentos no saludables" (Roberto et al., 2015). Estudios epidemiológicos han evidenciado una asociación positiva entre adiposidad, marcadores metabólicos y de inflamación, los que acrecientan el riesgo de enfermedades metabólicas, diabetes mellitus tipo 2 y cardiovasculares (Labraña et al., 2017). Al igual, no se realizan actividades físicas que demanden un gasto energético significativo que contrarresten el alto índice calórico. Las anteriores atenuantes descritos conllevan a demarcar un panorama desalentador y nocivo, tanto para la salud como para la calidad vida de la población colombiana.

En este contexto, los estudiantes universitarios como población adulta son una población heterogénea, evidenciando un aumento progresivo del sobrepeso y obesidad, asociadas a factores de riesgo cardiovascular (Hassapidou et al., 2013). Además, los estudiantes universitarios tienen una percepción regular sobre sus conocimientos acerca de hábitos de alimentación saludable, actividad física y control de peso (Rodríguez, Restrepo y Deossa, 2016). Por otra parte, los diversos factores que determinan el sobrepeso o la obesidad pueden ser contrarrestados con el ejercicio físico definido según Escalante (2011), como: "la actividad física planificada, estructurada y repetida, cuyo objetivo es adquirir, mantener y/o mejorar la condición física" (p. 326).

En este sentido, la variedad de métodos de entrenamiento, de actividad física y de ejercicio, permiten generar una alternativa denominada entrenamiento concurrente, considerada como una estrategia que combina la resistencia aeróbica y la fuerza, en el mismo período de sesiones (Bell, Syrotuik, Martín, Burnham, \& Quinney, 2000), y que están condicionados por la manipulación de los componentes o parámetros que definen la dosis del estímulo aplicado (volumen, intensidad, densidad, frecuencia, $y$ 
metodología y por la forma de combinar o secuenciar los entrenamientos de cada capacidad (García-Orea, Elvar, Campillos, Grigoletto, \& Del Rosso, 2016). Así, estas dos capacidades físicas básicas sometidas al trabajo periodizado, con intensidades de cargas de trabajo progresiva permiten mejoras en la condición física general, específicamente, mediante los procesos fisiológicos vitales. Con referencia a las adaptaciones fisiológicas de la fuerza, donde se produce un incremento de la fuerza contráctil máxima y posibles adaptaciones morfológicas que incluyen hiperplasia, cambios en el tipo de fibra, arquitectura muscular, densidad de miofilamento y la estructura del tejido conectivo y los tendones (Folland \& Williams, 2007). Por el contrario, las adaptaciones producidas por el ejercicio aeróbico se manifiestan en el incremento de la densidad mitocondrial y la capacidad oxidativa y una menor producción de lactato durante el ejercicio en una intensidad determinada (Holloszy, 1967; Holloszy \& Coyle, 1984). Por consiguiente, el trabajo de estas capacidades físicas contrarresta diversos desórdenes que desmejoran la capacidad funcional y la salud metabólica, ayudan en la prevención y tratamiento de numerosas enfermedades durante el transcurso de la vida, las recomendaciones internacionales sobre ejercicio físico sugieren la práctica de ambos tipos de entrenamiento (Garber et al., 2011).

Por lo tanto, en distintos estudios se evidencia que el entrenamiento concurrente ayuda a contrarrestar o controlar los diferentes trastornos cardio-metabólicos asociados con el sobrepeso y la obesidad (Jakicic \& Otto, 2006), a mejorar y disminuir la masa corporal total y el IMC y el \% MG (Antunes et al., 2013; Pereira et al., 2018; García \& Galindo, 2017; Monteiro et al., 2015). Además, a reducir significativamente la grasa corporal, el IMC circunferencia abdominal, circunferencia de cadera, y circunferencia de cintura (Colato et al., 2014; Yánez et al., 2017; Sigal et al., 2014; Banitalebi \& Baghanari, 2015). Este entrenamiento también, mejora el perfil lipídico y presenta una reducción significativa en la masa corporal, el IMC y la masa grasa y el porcentaje de grasa corporal después de la intervención a corto y largo plazo de ejercicio concurrente (Browning, Bean, Wickham, Stern \& Evans 2015; Inoue et al., 2015). Además, de promover mejoras significativas en la composición corporal y las variables metabólicas, al disminuir la masa libre de grasa y la masa grasa del tronco (Albuquerque et al., 2014; Antunes et al., 2015; Cervantes \& Hernández, 2017). Otros estudios muestran resultados significativos en la mejora de la función física, la fuerza muscular y en el consumo máximo de oxígeno (Ferrari et al., 2016; De Farias, BorbaPinheiro, Oliveira, \& de Souza Vale, R., 2014; Brunelli, et al., 2015; García, Arriaza, Valverde, Moya, \& Mardones, 2017).

\section{MATERIALES Y MÉTODOS}

El diseño metodológico utilizado para esta investigación es preexperimental, presenta una intervención con un programa de 
entrenamiento concurrente y evaluaciones de pre y pos-test, de las variables dependientes de un solo grupo, cuyo grado de control es mínimo (Hernández, Fernández \& Baptista, 2014).

El estudio se desarrolló en la Universidad Santo Tomás seccional Tunja, con estudiantes de primer semestre de 2019-1. Muestra de tipo no probabilística, con sujetos elegidos de manera no aleatoria (Otzen \& Manterola, 2017). Participantes: 12 sujetos de género masculino, en edades de 18 a 22 años. Criterios de inclusión: IMC entre el rango de 25 a 34.9 kg/m2; Certificado médico que indique no ser portador de diabetes mellitus tipo II, ni enfermedades cardiovasculares y/o respiratorias y que puede realizar actividad física vigorosa, así como la firma del formato de consentimiento informado. El presente estudio fue realizado cumpliendo los protocolos de ética según decreto 008430 de 1993 del Ministerio de Salud.

\section{Mediciones y Procedimientos}

A todos los estudiantes se les explicó en qué consistió el estudio, cuál era su finalidad y para qué se les requirió, así mismo, tuvieron conocimiento de los procedimientos que se llevarían a cabo, antes y durante las pruebas y los procedimientos de seguridad, así como el carácter voluntario de cada sujeto, quienes firmaron un consentimiento informado para la aceptación en la participación de la investigación. Los participantes fueron valorados en el gimnasio de la universidad
Santo Tomás seccional Tunja, con la debida autorización de los directivos de la institución. Todo el proceso se realizó con las respectivas consideraciones éticas.

\section{Valoración perfil antropométrico}

Todas las mediciones se realizaron en horas de la mañana, a los participantes se les indicó que para las respectivas valoraciones del pre-test y post-test, debían cumplir con los siguientes parámetros: 1- no ingerir ningún alimento, ni bebida, 2- haber egestado, 3utilizar ropa cómoda (pantaloneta).

Así, para definir la caracterización de cada sujeto, se procedió a la toma de peso, se utilizó la báscula digital Tanita referencia Bc585f Fitscan, Para la determinar la talla se utilizó un tallímetro portátil SECA modelo 213. Con los datos que se obtuvieron (peso y talla), se calculó el IMC mediante la ecuación propuesta por Quetelet en 1832 (Eknoyan, 2008); IMC = peso [kg]/talla [m] ${ }^{2}$, para ubicar al sujeto con relación a la tabla de clasificación según la Sociedad Española para el Estudio de la Obesidad (SEEDO 2007). Para determinar el \% MG, se utilizó el Adipómetro referencia Slim Guide con rango $80 \mathrm{~mm}$., para la toma de los pliegues o panículos cutáneos y cinta métrica BMI para medir longitudes segmentales y se utilizó el protocolo de Castellanos (2014), se midieron los pliegues cutáneos (tricipital, subescapular, suprailíaco, abdominal, cuadricipital y medial o peroneal), se calculó el \% MG mediante la fórmula de 
Yuhasz (1974): \% MG (hombres) $=\left(\begin{array}{ll}\Sigma & 6\end{array}\right.$ pliegues $(\mathrm{mm}) \times 0,1051)+2,585$.

\section{Valoración de la Fuerza (1 RM)}

Se determinó, teniendo en cuenta, el test de Repeticiones hasta la Fatiga Total (RFT) para estimar 1 RM. En las ecuaciones que se utilizan las RFT, el peso desplazado ha sido establecido bien de forma aleatoria, o se ha buscado mover cargas que permiten llegar a 5 RM, 10 RM o 20 RM (Laguado et al., 2013). Por lo tanto, se realizó una prueba indirecta con pesas, para seis tipos de ejercicios: 1- Curl femoral, 2- Press plano con barra, 3- Curl biceps con barra, 4- Extensión de pierna, 5Jalón polea al pecho y Tríceps polea, para evaluar la fuerza de los grupos musculares de la parte superior (pectoral, dorsal ancho, bíceps y tríceps) e inferior (cuádriceps femoral y bíceps femoral). Por consiguiente, al obtener el peso levantado en $\mathrm{Kg}$ y el número RFT ejecutadas en cada ejercicio, se procedió a determinar 1 RM mediante la ecuación propuesta por Brzycki (1993): Peso levantado en $\mathrm{Kg} /(1,0278-(0,0278 \times$ repeticiones).

\section{Cálculo de la Frecuencia Cardiaca Máxima (FCM)}

Se determinó para cada sujeto la FCM y se calculó utilizando la ecuación de: Tanaka et al. (2001):

$\boldsymbol{F C M}=\mathbf{2 0 8 , 7 5}$ - (edad $\boldsymbol{x}$ 0,73). La FCM se utilizó como parámetro, y así, establecer dentro del programa de entrenamiento concurrente las intensidades de trabajo para el ejercicio aeróbico.

Todos los datos recogidos se registraron en el programa Excel para el procesamiento de los diferentes cálculos de las variables a intervenir.

Programa de entrenamiento concurrente, sus indicadores son la fuerza y la resistencia aeróbica. La duración del programa de entrenamiento concurrente fue de 10 semanas, realizando 3 sesiones por semana, alternando los días (lunes, miércoles $y$ viernes o en su defecto martes, jueves y sábado), con un tiempo de 60 minutos por sesión, distribuidos así, 5 minutos de calentamiento, 25 minutos de ejercicios de fuerza, luego 25 minutos de ejercicio aeróbico, y finalizando, con 5 minutos de elongación muscular.

Para el protocolo de entrenamiento de fuerza surgieron dos tipos de sesiones, la sesión 1 compuesto por tres ejercicios: Curl femoral, press plano con barra y curl bíceps con barra y la sesión 2 compuesta por los ejercicios de: Extensión de pierna, jalón polea al pecho y tríceps polea. La primera semana consistió en la adaptación y el aprendizaje de: cómo usar las máquinas y los pesos, al llegar a la sesión cada sujeto realizaba 5 minutos de calentamiento (movilidad articular), para proceder a realizar 25 minutos de trabajo de fuerza con ejercicios programados en la sesión 1, con una intensidad del 30\% de 1 RM, realizaron 3 series por cada ejercicio de 20 repeticiones, con una recuperación de 45 segundos entre series y de 1 minuto entre ejercicios. Luego, del período de adaptación, 
se ajustó la carga incrementando el peso y la intensidad de 1 RM y se disminuyeron las repeticiones para las siguientes semanas distribuidas así: semana $2-3$ al $50 \%$ de intensidad-15 repeticiones; semana 4-5 al $60 \%$ de intensidad- 12 repeticiones y de la semana 6-10 al 75\% de intesidad-10 repeticiones.

El protocolo de entrenamiento aeróbico consistió en realizar 25 minutos por sesión, después del entrenamiento de fuerza, con una intensidad del $50 \%$ de la FCM en la primera semana de adaptación. Después se ajustaron las intensidades de acuerdo con los valores de la FCM, para las siguientes semanas distribuidas así: semana 2-3 al 60\% de la FCM; semana $4-5$ al $70 \%$ de la FCM; semana 6-10 al 80\% de la FCM y fue realizado en máquina trotadora T60 Visión Fitness o bicicleta spinning techno life evolution y la intensidad de ejercicio aeróbico se monitorizó usando un reloj Polar Ft1 monitor cardíaco.

\section{Diseño estadístico}

La presentación del análisis estadístico inicial se expone mediante un análisis de tipo descriptivo, donde se evidencian los principales rasgos de las variables a usar. Uno de los objetivos es establecer las diferencias que otorga este tratamiento, lo que condujo al uso de una prueba de: Shapiro Wilk, que presenta utilidad a causa del tamaño de la muestra $(n \leq 30)$ y permite determinar la normalidad de los datos, para el caso de este estudio el nivel de significancia nos evidenció normalidad en los datos. A raíz de este supuesto, se trabajó con pruebas de tipo paramétrico, que asumen distribuciones estadísticas subyacentes a los datos en este caso normal. La prueba paramétrica que se usó permite mostrar, sí, existen diferencias significativas entre el antes y el después, o identificar los efectos de la intervención. Para determinar la significancia estadística se estableció un nivel de confianza del 95\% ( $p<$ 0.05). Para la tabulación y el análisis de los datos se utilizaron dos softwares Excel 2013 (Albuquerque, Estados Unidos) y SPSS 2016 Versión 24.0.

\section{RESULTADOS}

Los individuos fueron clasificados en sobrepeso grado II (75\%) y obesidad Tipo I (25\%). La información que proporciona la tabla número 1, permite referir las variables que se utilizaron para el cálculo del porcentaje de masa, evidenciando medidas de tendencia central y variabilidad. Son variables de tipo cuantitativo discretas, que pertenecen a la escala de medición, por esta razón, estas presentan valores mínimos y máximos en el rango de la escala de medición asignada; con respecto a la desviación estándar, se observa que las variables tienen poca dispersión, resultado que favorece inicialmente la interpretación de la información. Además, que no se presentaron valores perdidos o que no corresponden de acuerdo con los rangos establecidos. No se presentan problemas de asimetría, ni de curtosis. 
Tabla 1. Concentrado de los estadísticos descriptivos para las variables intervinientes.

\begin{tabular}{cccc}
\hline MEDIDA & EDAD & PESO (Kg) & TALLA (mts) \\
\hline Promedio: & 19,75 & 83,13 & 1,70 \\
Desviación: & 1,86 & 8,38 & 0,04 \\
Mínimo: & 18,00 & 69,70 & 1,64 \\
Máximo: & 22,00 & 95,50 & 1,78 \\
Asimetrías: & 0,33 & $-0,15$ & 0,15 \\
Curtosis: & $-2,05$ & $-0,75$ & $-0,57$ \\
\hline
\end{tabular}

La tabla 2. Presenta los valores de las variables antes y después de la intervención para el conjunto de los 12 individuos. De igual forma, se presentan los promedios y las desviaciones para cada una de ellas, donde se evidencia una disminución en el promedio de las variables de peso, IMC y en él \% MG. Además, se evidencia un aumento en la variable de fuerza tanto en el tren inferior como en el superior.

Tabla 2. Concentrado de los estadísticos descriptivos de pre-test y pos-test de las variables. intervinientes

\begin{tabular}{ccc}
\hline VARIABLE & PRE-TEST & POS-TEST \\
\hline IMC: & $28,67 \pm 2,7$ & $28,18 \pm 2,38$ \\
\% MG: & $18,4 \pm 3,84$ & $14,35 \pm 2,78$ \\
Peso Kg: & $83,13 \pm 8,38$ & $81,3 \pm 7,54$ \\
Fuerza 1 RM Kg: & & \\
Tren inferior: & $71,75 \pm 29,17$ & $100,08 \pm 37,64$ \\
Tren superior: & $48 \pm 22,68$ & $59,95 \pm 22,93$ \\
\hline
\end{tabular}

\section{ANÁLISIS INFERENCIAL}

En primera instancia: Se identificó la distribución que sigue las variables objeto, que para este estudio son: el IMC, IMCpre y IMCpost, suma pliegues cutáneos (sumapre y sumapost), \% MG (porcepre y porcepost) y para la distribución de la variable peso (pesopre y pesopost, tanto en el proceso antes y después de la intervención. Igualmente, la distribución de las variables de fuerza constituida por los ejercicios de Extensión de pierna, Curl Femoral, Press plano con barra, Curl bíceps con barra, Jalón polea al pecho y Triceps polea de pre y post. Dado que el tamaño de la muestra es menor ( $n \leq 30)$, se aplica el estadístico de ShapiroWilk, cuyas hipótesis son: 


\section{$\mathrm{H}_{0}$ : Los datos de la variable IMC (IMCpre ó IMCpost) siguen distribución normal $\mathrm{H}_{1}$ : Los datos de la variable IMC (IMCpre ó IMCpost) siguen distribución normal}

La prueba se estimó con un nivel de significancia del 5\%, para las dos variables el p-valor (Sig.) mostrado en la tabla 3 indica que las variables tienen valores mayores al nivel de significancia, por eso, se acepta la hipótesis nula $\left(\mathrm{H}_{\mathrm{o}}\right)$, porque, se tiene que los datos para las variables siguen una distribución normal en el pre test y pos test, excepto la variable para el ejercicio de extensión de pierna que los p-valor son menores al nivel de significancia, por lo tanto, los datos no siguen una distribución normal. Para las demás variables indica, que los datos se pueden analizar bajo pruebas paramétricas, para hacer inferencia sobre el efecto del programa de entrenamiento concurrente de 10 semanas, que es aplicado a los individuos de la muestra.

Tabla 3. Estadístico de Shapiro- Wilk para variables del grupo.

\begin{tabular}{ccccc}
\hline & \multicolumn{3}{c}{ Shapiro-Wilk } \\
\hline Variable & & Estadístico & $\begin{array}{c}\text { Grados de } \\
\text { libertad }\end{array}$ & Sig. \\
\hline Índice de masa corporal: & Pretest &, 914 & 12 &, $\mathbf{2 3 7}$ \\
Índice de masa corporal: & Postest &, 884 & 12 &, 099 \\
Suma pliegues cutáneos: & Pretest & 0,94 & 12 & $\mathbf{0 , 4 9 8}$ \\
Suma pliegues cutáneos: & Postest & 0,944 & 12 & $\mathbf{0 , 5 4 7}$ \\
\% MG: & Pretest & 0,94 & 12 & $\mathbf{0 , 4 9 8}$ \\
\% MG: & Postest & 0,944 & 12 & $\mathbf{0 , 5 4 7}$ \\
Peso: & Pretest & 0,949 & 12 & $\mathbf{0 , 6 2 0}$ \\
Peso: & Postest & 0,933 & 12 & $\mathbf{0 , 4 0 8}$ \\
Curl femoral: & Pretest & 0.979 & 12 & $\mathbf{0 . 9 8 0}$ \\
Curl femoral: & Postest & 0.945 & 12 & $\mathbf{0 . 5 6 4}$ \\
Press plano: & Pretest & 0.938 & 12 & $\mathbf{0 . 4 7 5}$ \\
Press plano: & Postest & 0.978 & 12 & $\mathbf{0 . 9 7 2}$ \\
Curl de bíceps: & Pretest & 0.965 & 12 & $\mathbf{0 . 8 4 9}$ \\
Curl de bíceps: & Postest & 0.973 & 12 & $\mathbf{0 . 9 4 4}$ \\
Extensión de pierna: & Pretest & 0.844 & 12 & $\mathbf{0 . 0 3 1}$ \\
Extensión de pierna: & Postest & 0.825 & 12 & $\mathbf{0 . 0 1 8}$ \\
Jalón polea al pecho: & Pretest & 0.975 & 12 & $\mathbf{0 . 9 5 2}$ \\
Jalón polea al pecho: & Postest & 0.931 & 12 & $\mathbf{0 . 3 9 0}$ \\
\hline
\end{tabular}


PRUEBAS T- PARA MUESTRAS RELACIONADAS.

Con un nivel de significancia del 5\%, se buscan evaluar las siguientes hipótesis:

\begin{tabular}{|c|c|}
\hline $\begin{array}{l}H_{0}: \text { IMCPRE}=I M C P O S T \\
H_{1}: \text { IMCPRE } \neq \text { IMCPOST }\end{array}$ & $\begin{array}{l}\mathrm{H}_{0}: \text { PORCPRE }=\text { PORCPOST } \\
\mathrm{H}_{1}: \text { PORCPRE } \neq \text { PORCPOST }\end{array}$ \\
\hline $\mathrm{H}_{0}:$ SUMAPRE=SUMAPOST & $\mathrm{H}_{0}:$ PESOPRE=PESOPOST \\
\hline $\mathrm{H}_{1}:$ SUMAPRE $\neq$ SUMAPOST & $\mathrm{H}_{1}:$ PESOPRE $\neq$ PESOPOST \\
\hline $\mathrm{H}_{0}:$ CURLFEMPRE=CURLFEMORPOST & $\mathrm{H}_{0}:$ PRESSPLPRE=PRESSPLPOST \\
\hline $\mathrm{H}_{1}:$ CURLFMORPRE $\neq$ CURLFMORPOST (1) & $\mathrm{H}_{1}:$ PRESSPLPRE $\neq$ PRESSPLPOST (2) \\
\hline $\begin{array}{c}\mathrm{H}_{0}: \text { CURLBICPRE=CURLBIPOST } \\
\mathrm{H}_{1}: \text { CURLBICPRE } \neq \text { CURLBIPOST }(3)\end{array}$ & $\begin{array}{c}\mathrm{H}_{0}: \text { JALÓNPPRE }=\text { JALÓNPPOST } \\
\mathrm{H}_{1}: \text { JALÓNPPRE } \neq \text { JALÓNPPOST (4) }\end{array}$ \\
\hline \multicolumn{2}{|c|}{$\begin{array}{c}\mathrm{H}_{0}: \text { TRICEPRE }=\text { TRICEPOST } \\
\mathrm{H}_{1}: \text { TRICEPOPRE } \neq \text { = TRICEPOST }(5)\end{array}$} \\
\hline
\end{tabular}

En la siguiente tabla, se presenta el nivel de significancia de la prueba T, para cada una de las siguientes hipótesis:

Tabla 4. Estadístico prueba $T$ para muestras relacionadas.

\begin{tabular}{llccccccc}
\hline & Media & $\begin{array}{c}\text { Desv. } \\
\text { Desviación }\end{array}$ & $\begin{array}{c}\text { Desv. Error } \\
\text { promedio }\end{array}$ & $\begin{array}{c}95 \% \text { de intervalo de confianza } \\
\text { de la diferencia } \\
\text { Inferior }\end{array}$ & Superior & T & Sig. \\
(Bilateral)
\end{tabular}

En la tabla 4: Se evidencia que no hay cambios significativos para las variables IMC y para el peso, teniendo en cuenta, que los p-valores (Sig.) son mayores al nivel de significancia de $5 \%$. Por el contrario, en las variables de suma de pliegues cutáneos y la variable de \% MG, muestran un cambio estadísticamente significativo, porque el $\mathrm{p}$ valor de las dos variables son menores al nivel de significancia de $5 \%$, así, se concluye que, después de aplicar un programa de entrenamiento concurrente de 10 semanas de intervención, se produce un cambio significativo en las variables de suma de pliegues, y, por tanto, en el \% MG de los 12 individuos de la muestra.

Para el análisis de la variable de fuerza a continuación se presenta un análisis 
inferencial de comparación de medias: Mediante una prueba T., para cada una de las variables que se distribuyen normalmente en cada uno de los ejercicios de Curl Femoral, Press plano con barra, Curl bíceps con barra, Jalón polea al pecho y Triceps polea aplicados en la intervención.

En la tabla 5: Se presenta los p-valores de la prueba $\mathrm{T}$ para muestras relacionadas, para las anteriores hipótesis.

Tabla 5. Estadístico prueba T para muestras relacionadas.

\begin{tabular}{|lccccccccc}
\hline \multicolumn{7}{c}{ Diferencias emparejadas } \\
\hline & Media & Desviación & $\begin{array}{c}\text { Desv. Error } \\
\text { promedio }\end{array}$ & $\begin{array}{c}95 \% \text { de intervalo de } \\
\text { confianza de la diferencia } \\
\text { Inferior }\end{array}$ & Superior & T & gl & Sig. \\
(bilateral)
\end{tabular}

Todos los p-valores (Sig.) de la tabla 5, para las variables muestran un cambio significativo, debido a que, da un valor de $\mathrm{p}<0,05$, por lo cual, se acepta la hipótesis alternativa $H_{1}$, para todas la hipótesis (par 1 , 2, 3, 4 y 5), por lo tanto, para todos los individuos de la muestra, si, hubo un cambio significativo en el aumento de 1 RM, luego que se les aplicara el programa de entrenamiento concurrente.

\section{DISCUSIÓN}

A partir de los resultados del presente estudio, fue posible verificar los efectos del programa de entrenamiento concurrente, que promovió cambios beneficiosos y significativos en el grupo de intervención, observando una reducción en el \% MG (Pre: 18,4 $\pm 3,84$ / Post: 14,35 $\pm 2,78 p<0,000$ ). Aunque, con una reducción en el IMC que no fue estadísticamente significativa (Pre: 28,67 $\pm 2,7$ / Post: $28,18 \pm 2,38 p<0,087$ ). Además, mejoró la FM del tren inferior (Pre: 71,75 \pm 29,17 / Post: 100,08 $\pm 37,64$, aumentando el $39,5 \%$ ) y en el tren superior mejoró (Pre: $48 \pm$ $22,68 / 59,95 \pm 22,93$, aumentando el 24,9\%) de 1 RM de los diferentes ejercicios ejecutados con un valor de significancia de $(p$ $<0,05)$.

En distintos estudios se evidencia que el entrenamiento concurrente ayuda a contrarrestar o controlar los diferentes trastornos cardio-metabólicos asociados con el sobrepeso y la obesidad (Jakicic \& Otto, 
2006). En un estudio realizado por: Antunes et al. (2013), en adolescentes obesos sometidos a un protocolo de entrenamiento combinado de ejercicios de fuerza y ejercicio aeróbico, los autores observaron una reducción significativa en el \% MG $(p<0,001)$ y en el IMC hubo una reducción no significativa ( $p$ $<0,350$ ), después de 20 semanas de intervención, de resultados similares en nuestro estudio, donde también, se redujo el \% MG. Sin embargo, hubo reducción en el IMC, pero no fue estadísticamente significativo después de 10 semanas. Por otra parte, el mismo autor y colaboradores en 2015 analizaron los efectos de un programa de intervención de entrenamiento concurrente de 20 semanas, con un protocolo de ejercicios, que se basó en sesiones de 30 minutos de actividades aeróbicas (caminar y trotar), seguidas de 30 minutos de ejercicios de fuerza, tres veces por semana, el grupo interviniente dividido en ambos géneros. Evidenciando, reducciones en el IMC ( $p$ $<0,033$ ) hombres y mujeres ( $p<0,004$ ), estos resultados sugieren que el tipo de entrenamiento que utilizaron pudo inferir en estos resultados. Contrario al protocolo que se realizó en el presente estudio, donde se realizó primero: el trabajo de fuerza, y luego, de ejercicios aeróbicos durante 10 semanas, no se dió la reducción del IMC, pero, si, aumentó significativamente la FM.

Otros estudios realizados en poblaciones con sobrepeso y obesidad, donde se aplicaron programas de entrenamiento concurrente a 12 y 15 semanas, observaron una reducción significativa en el peso $(p=$ $0.015)$, el IMC $(p=0.011)$ y el porcentaje de masa grasa $(p=0.013)$, en la $\Sigma 4$ pliegues cutáneos $(p=0,040)$, después de la intervención (Colato et al., 2014; Yánez et al., 2017). En este contexto, otros estudios, donde el tiempo de duración fue de 12 y 20 semanas, en los que se evaluaron los efectos del entrenamiento concurrente sobre la obesidad, lograron establecer la reducción de $\%$ de grasa total, en un estudio fue $(-2,2 \%$ a 0,2\%) y en otro de (-2,9\% y -3,6\%) (García \& Gil, 2017; Monteiro et al., 2015), en línea con nuestro estudio, donde disminuyó el \% MG como indicador en la reducción de la grasa total en el grupo de jóvenes. No obstante, parece ser que el tiempo de duración en la aplicación del entrenamiento concurrente es un factor determinante, para conseguir mejoras significativas en todas las variables estudiadas, lo que nos lleva a pensar que 10 semanas realizadas en este estudio no fueron suficientes para modificar todas las variables.

Según otro estudio realizado por Varela (2014), donde compararon la efectividad de un programa de entrenamiento concurrente de 8 semanas de duración en jóvenes universitarios, con cargas de trabajo al 65 a $85 \%$ de la FCM y fuerza con cargas medias al $40 \%$ a $50 \%$ de 1 RM, los resultados obtenidos presentan una reducción no significativa, ni para el peso corporal $(p=0,491)$, ni para la $\Sigma 4$ Pliegues cutáneos $(p=0,422)$, ni para el IMC $(p=0,3)$. Sin embargo, en el estudio que se realizó, se presentó un cambio significativo en la $\Sigma 6$ Pliegues cutáneos ( $p<0,000$ ), también, se evidenció que hubo una reducción, que no fue estadísticamente significativa en el peso ( $p<0,082)$, ni en el IMC ( $p<0,087)$, otro estudio con una duración de 8 semanas realizado por: 
Banitalebi \& Baghanari (2015), sobre la composición corporal en mujeres, el entrenamiento no influyó en la respuesta adaptativa en las variables intervenidas, tanto para el porcentaje de grasa corporal $(P=$ 0.08), ni en la fuerza máxima de la extremidad superior $(P=0.07)$, por el contrario, en nuestro estudio, si, hubo un cambio significativo en el aumento de la FM del tren superior $(p<0,05)$. Sin embargo, hubo diferencias significativas en la fuerza máxima del miembro inferior $(P=0,000)$ coincidiendo con este estudio del tren inferior $(p<0,05)$, deduciendo que la cantidad de ejercicios ejecutados en la investigación realizada permitió un cambio significativo en ambas extremidades. En comparación del estudio realizado por: Sigal et al. (2014) que con una mayor población 78 sujetos y con una intervención de 24 semanas, donde se aplicaron el entrenamiento combinado obtuvieron una reducción moderada en el \% MG y en el IMC, lo que evidencia que el tiempo de duración debe ser mayor a 10 semanas.

En el estudio de Varela (2014), también, evaluó la fuerza y que fue estadísticamente significativo para 1 RM estimada, tanto en el ejercicio de press banca, como en la media sentadilla ( $p=0,000$ para ambos), lo que coincide con los ejercicios ejecutados en nuestro estudio. Donde aumenta 1 RM en 5 de los 6 ejercicios Curl Femoral, Press plano con barra, Curl bíceps con barra, Triceps polea $(p<0,000)$ y Jalón polea al pecho $(p=$ $0,043)$, mejorando la fuerza muscular. En este sentido: De Farias et al. (2014) evaluaron los Efectos de un Programa de Entrenamiento Concurrente de 12 semanas de duración, sobre la fuerza muscular en mujeres mayores, evidenciando que la fuerza de los miembros inferiores mejoró $(p<0,05)$, equivalente a un aumento del $37.7 \%$ y la fuerza tren superior incrementó $(p<0,05)$, equivalente a un aumento del 30,56 \% después del entrenamiento. Un incremento también evidenciado en el estudio realizado, donde aumentó la fuerza muscular en un $39,5 \%$ tren inferior y $24,9 \%$ para el tren superior. Observamos que la fuerza muscular, si, aumenta en programas a corta duración a partir de 8 semanas; lo que indicaría, hipertrofia de la fibra muscular, debido al incremento de la sección transversal del músculo, y a una mayor síntesis proteica, así como a la eficiencia de la activación de unidades motoras (Fry, 2004; Wilmore \& Costill, 2010).

El entrenamiento concurrente ha sido una alternativa para mejorar la composición corporal y la condición física general de las personas con patologías como el sobrepeso y la obesidad y que demuestra que, si bien los cambios generados en algunos casos no son significativos estadísticamente, si causan unos cambios y respuestas adaptativas a nivel fisiológico y que el tiempo de duración debería ser mayor de 10 semanas, para optimizar los resultados de las diferentes variables a investigar. Además, estudios como el de: Browning et al. (2015), con un programa de entrenamiento combinado y con 24 semanas de ejecución, se observó una disminución en el peso corporal (-4.50 \pm 3.53 $\mathrm{kg}, \mathrm{p}<.001)$, masa grasa $(-4.50 \pm 2.20 \mathrm{~kg}$, $\mathrm{p}<.001)$ y porcentaje de grasa corporal ($2.97 \% \pm 1.45 \%, p<.001)$. 
Por lo tanto, un periodo prolongado de entrenamiento concurrente podría ocasionar cambios significativos. Además, promover las mejoras en la composición corporal y las variables metabólicas, al disminuir la masa libre de grasa y la masa grasa del tronco, al terminar con 16 semanas de intervención (Albuquerque et al., 2014; Cervantes \& Hernández, 2017). Además, de mejorar la función física, la fuerza muscular, y al disminuir significativamente en el porcentaje de grasa corporal; mejora la potencia muscular y la calidad muscular, después de 12 y 24 semanas respectivamente (Brunelli et al., 2015; Ferrari et al., 2016). Estos hallazgos sugieren que esta población con sobrepeso y obesidad requieren de un tiempo más prolongado, por ende, mayor a 10 semanas de intervención.

\section{CONCLUSIONES}

Los resultados del presente estudio, sugieren que 10 semanas de entrenamiento concurrente, tres veces por semana y 60 minutos por sesión con ejercicios de fuerza combinada, con ejercicio aeróbico se consiguieron reducir el porcentaje de masa grasa, mejorando el perfil antropométrico. Aunque, el índice de masa corporal y el peso se redujo no mostraron cambios estadísticamente significativos. Con relación a la fuerza los resultados logrados muestran estadísticamente un aumento significativo de la fuerza muscular del tren inferior y superior en los adultos jóvenes con sobrepeso u obesidad. Según el entrenamiento concurrente que se desarrolló, este estudio sugiere aplicar en la sesión, primero el trabajo de fuerza, y luego, resistencia aeróbica, para que los depósitos de glucógeno en el músculo se degraden y la contribución de los ácidos grasos se incrementen con el objetivo de perder grasa corporal (Zussa, 2017).

\section{LIMITACIONES DEL ESTUDIO}

La ausencia de un grupo control en la investigación que permitiera la comparación de las diferentes variables del grupo intervenido. Además, como la medición de pliegues cutáneos se puede presentar un grado de error en sus estimaciones, dado que la manipulación fue realizada por el investigador, quien no cuenta con acreditación ISAK. Sin embargo, la toma de pliegues cutáneos es un método fácil de usar en una investigación de campo. Además, la ecuación de Quetelet para estimar el IMC como predictor de acumulación de masa grasa, muestra una baja confiabilidad para estimar adiposidad a nivel individual, particularmente, en hombres y cuando el IMC es menor de $30 \mathrm{~kg} / \mathrm{m}^{2}$ (Carrasco, Reyes, Rímler, \& Ríos, 2004).

También, la ausencia de una prueba de $\mathrm{VO}_{2 \max }$ en el momento de valoración para observar los posibles efectos producidos por el ejercicio aeróbico. Y, por último, no se realizó una intervención nutricional supervisada, solo fue recomendaciones nutricionales de manera escrita, por cuanto 
es difícil que los participantes cumplieran con este parámetro a cabalidad, son posibles factores de confusión o de una mejor interpretación de los resultados obtenidos. Sin embargo, este estudio consideró la individualidad de los sujetos en las diferentes pruebas y aplicaciones del ejercicio en el estudio.

\section{REFERENCIAS BIBLIOGRÁFICAS}

ALBUQUERQUE, N., REBOUÇAS, G., MATOS, V., SALGUEIRO, C., KNACKFUSS, M. \& MEDEIROS, H. 2014. Efecto del Entrenamiento Concurrente en la Composición Corporal y Perfil Lipídico en Adolescentes con Sobrepeso. JEPonline,17(6):34-44.

ANTUNES, B., CHRISTOFARO, D., MONTEIRO, P., SILVEIRA, L., FERNANDES, R., MOTA, J., y FREITAS, I. 2015. Efecto del entrenamiento concurrente sobre variables bioquímicas específicas de género y adiposidad en adolescentes obesos. Archivos de endocrinología y metabolismo, 59 (4), 303-309.

ANTUNES, B., MONTEIRO, P., SILVEIRA, L., CAYRES, S., SILVA, C., JÚNIOR, F., \& FORTE, I. 2013. Efecto del entrenamiento simultáneo sobre los factores de riesgo y la esteatosis hepática en adolescentes obesos. Revista Paulista de Pediatría, 31 (3), 371-376.

BANITALEBI, E. y BAGHANARI, H. 2015. Efecto del orden de secuencia del entrenamiento combinado (resistencia y resistencia) sobre la fuerza, la capacidad aeróbica y la composición corporal en mujeres mayores. Middle East Journal of Rehabilitation and Health ,2 (2).

BELL, G., SYROTUIK, D., MARTÍN, T., BURNHAM, R. y QUINNEY, H. 2000. Efecto del entrenamiento concurrente de fuerza y resistencia sobre las propiedades del músculo esquelético y las concentraciones hormonales en humanos. Revista europea de fisiología aplicada, 81 (5), 418-427.

BROWNING, M., BEAN, M., WICKHAM, E., STERN, M. y EVANS, R. 2015. Mejoras cardiometabólicas y de estado físico en niñas obesas que aumentaron o perdieron peso durante el tratamiento. The Journal of pediatrics, 166 (6), 1364-1369.

BRUNELLI, D., CHACÓN, M., GÁSPARI, A., LOPES, W., BONGANHA, V., BONFANTE, I. L., \& CAVAGLIERI, C. 2015. Combined Training Reduces Subclinical Inflammation in Obese Middle-Age Men. Medicine and science in sports and exercise, 47(10), 2207-2215.

BRZYCKI, M. 1993. Strength testing-predicting a one-rep max from reps-to-fatigue. Journal of Physical Education, Recreation \& Dance, 64(1), 88-90. 
CARRASCO, N., REYES, E., RIMLER, S., \& RÍOS, F. 2004. Exactitud del índice de masa corporal en la predicción de la adiposidad medida por impedanciometría bioeléctrica. Archivos latinoamericanos de nutrición, 54(3), 280-286.

CERVANTES, J., \& HERNÁNDEZ, J. 2017. Effect of High-Intensity and Concurrent Training in Body Composition in Cos-ta Rican Overweight and Obese Women. Arch Sports Med, 1(2), 65-74. COLATO, A., ABREU, F., MEDEIROS, N., LEMOS, L., DORNELES, G., RAMIS, T., \& PERES, A. 2014. Efectos del entrenamiento concurrente sobre los marcadores inflamatorios y la expresión de CD4, CD8 y HLA-DR en adultos con sobrepeso y obesos. Diario de la ciencia y el ejercicio físico, 12 (2), 55-61.

DE FARÍAS, M., BORBA, C., OLIVEIRA, M., \& DE SOUZA, R. 2014. Efectos de un programa de entrenamiento concurrente sobre la fuerza muscular, flexibilidad y autonomía funcional de mujeres mayores. Ciencias de la Actividad Física, 15(2), 13-24.

ESCALANTE, Y. 2011. Actividad física, ejercicio físico y condición física en el ámbito de la salud pública. Revista Española de Salud Pública, 85(4), 325-328.

EKNOYAN, G. 2008. Adolphe Quetelet (1796-1874). The average man and indices of obesity. Nephrol Dial Trasplant, 23, 47-51.

FERRARI, R., FUCHS, S., KRUEL, L., CADORE, E., ALBERTON, C., PINTO, R., \& UMPIERRE, D. 2016. Effects of different concurrent resistance and aerobic training frequencies on muscle power and muscle quality in trained elderly men: a randomized clinical trial. Aging and disease, 7(6), 697.

FOLLAND, P., \& WILLIAMS G. 2007. Las adaptaciones al entrenamiento de fuerza. Medicina deportiva. 37: 145.

GARBER, C., BLISSMER, B., DESCHENES, M., FRANKLIN, B., LAMONTE, M., LEE, I., Y SWAIN, D. 2011. Colegio Americano de Medicina Deportiva puesto de posición. Cantidad y calidad del ejercicio para desarrollar y mantener la condición cardiorrespiratoria, músculo-esquelética y neuromotora en adultos aparentemente sanos: Guía para la prescripción de ejercicios. Medicina y Ciencia en el Deporte y el Ejercicio, 43 (7), 1334-1359.

GARCÍA, J., \& GALINDO, P. 2017. Un programa de entrenamiento dirigido a la pérdida de peso: Uso del entrenamiento concurrente. E-motion: Revista de Educación, Motricidad e Investigación, (9), 42-64

GARCÍA-MANSO, J., ARRIAZA-ARDILES, E., VALVERDE, T., MOYA-VERGARA, F., \& MARDONESTARE, C. 2017. Efectos de un entrenamiento concurrente de fuerza y resistencia sobre carreras de media distancia. Cultura, Ciencia y Deporte, 12(36), 221-227.

GARCÍA-OREA, G., ELVAR, J., CAMPILLOS, J., GRIGOLETTO, M., \& DEL ROSSO, S. 2016. Entrenamiento Concurrente de Fuerza y Resistencia: Una Revisión Narrativa. Int J Phys Exerc Heal Sci Trainers, 1.

HASSAPIDOU, M., PAPADOPOULOU, S., VLAHAVAS, G., KAPANTAIS, E., KAKLAMANOU, D., PAGKALOS, I., Y TZOTZAS, T. 2013. Asociación de actividad física y patrones de estilo de vida 
sedentarios con obesidad y comorbilidades cardio-metabólicas en adultos griegos: Datos de la Encuesta Nacional de Epidemiología. Hormonas, 12 (2), 265-274.

HERNÁNDEZ, S., FERNÁNDEZ, C., \& BAPTISTA, B. 2014. Metodología de la Investigación. México: Editorial McGraw-Hill.

HOLLOSZY, J. (1967). Adaptaciones bioquímicas en los efectos musculares del ejercicio sobre la captación de oxígeno mitocondrial y la actividad de las enzimas respiratorias en el músculo esquelético. Diario de química biológica, 242 (9), 2278-2282.

HOLLOSZY, J., \& COYLE, E. 1984. Adaptaciones del músculo esquelético al ejercicio de resistencia y sus consecuencias metabólicas. Revista de fisiología aplicada, 56 (4), 831-838.

JAKICIC, J. y OTTO, A. 2006. Tratamiento y prevención de la obesidad: ¿Cuál es el papel del ejercicio?: Revisiones nutricionales, 64 (supl_1), S57-S61.

INOUE, D., DE MELLO, M., FOSCHINI, D., LIRA, F., GANEN, A., CAMPOS, R., y TUFIK, S. 2015. La fuerza periodizada lineal y ondulada más el entrenamiento aeróbico promueven beneficios similares y conducen a una mejora de la resistencia a la insulina en adolescentes obesos. Diario de la diabetes y sus complicaciones, 29 (2), 258-264.

LABRAÑA, A., DURÁN, E., MARTÍNEZ, M., LEIVA, A., GARRIDO-MÉNDEZ, A., DÍAZ, X., \& CELISMORALES, C. 2017. Menor peso corporal, de índice de masa corporal y de perímetro de cintura se asocian a una disminución en factores de riesgo cardiovascular en Población Chilena: Findings from the Chilean health surveyç. Revista médica de Chile, 145(5), 585-594.

MARTíN, A. 2014. Estudio sobre el perfil antropométrico, la composición corporal y el somatotipo en pacientes con síndrome coronario agudo en el área de salud de Cáceres (Doctoral dissertation, Universidad Complutense de Madrid).

MONTEIRO, P., CHEN, K., LIRA, F., SARAIVA, B., ANTUNES, B., CAMPOS, E., \& FREITAS, I. 2015.

Concurrent and aerobic exercise training promote similar benefits in body composition and metabolic profiles in obese adolescents. Lipids in health and disease, 14(1), 153.

ORGANIZACIÓN MUNDIAL DE LA SALUD. 2017. Enfermedades no transmisibles. Recuperado el 30 de noviembre de 2017. http://www.who.int/es/news-room/fact-

sheets/detail/noncommunicable-diseases

ORGANIZACIÓN MUNDIAL DE LA SALUD. 2018. Obesidad y sobrepeso. Recuperado el de 16 de febrero de 2018. http://www.who.int/es/news-room/fact-sheets/detail/obesity-andoverweight.

OTZEN, T., \& MANTEROLA, C. 2017. Técnicas de Muestreo sobre una Población a Estudio. International Journal of Morphology, 35(1), 227-2.

PEREIRA, A., SANTOS, G., BAGANHA, R., DE OLIVEIRA, J., HARLEY, A., y OLIVEIRA, R. 2018. Efectos del Entrenamiento Aeróbico versus Entrenamiento de Resistencia en la Composición Corporal y los Parámetros Bioquímicos Sistémicos de Adultos con Sobrepeso u Obesos. Journal of Exercise Physiology Online, 21 (2), 227-240. 
ROBERTO, C., SWINBURN, B., HAWKES, C., HUANG, T., COSTA, S., ASHE, M. Y BROWNELL, K. 2015. Progreso irregular en la prevención de la obesidad: Ejemplos emergentes, barreras arraigadas y nuevas formas de pensar. The Lancet, 385 (9985), 2400-2409.

RODRÍGUEZ-ESPINOSA, H., RESTREPO-BETANCUR, L., \& DEOSSA-RESTREPO, G. 2016. Conocimientos y prácticas sobre alimentación, salud y ejercicio en universitarios de Medellín, Colombia. Perspectivas en Nutrición Humana, 17(1), 36-54.

SALAS-SALVADÓ, J., RUBIO, M., BARBANY, M., MORENO, B., \& DE LA SEEDO, G. 2007. Consenso Seedo 2007 para la evaluación del sobrepeso y la obesidad y el establecimiento de criterios de intervención terapéutica. Medicina clínica, 128(5), 184-196.

SIGAL, R., ALBERGA, A., GOLDFIELD, G., PRUD'HOMME, D., HADJIYANNAKIS, S., GOUGEON, R., \& WELLS, G. 2014. Efectos del entrenamiento aeróbico, el entrenamiento de resistencia, o ambos, sobre el porcentaje de grasa corporal y los marcadores de riesgo cardio-metabólico en adolescentes obesos: la alimentación saludable aeróbica y el entrenamiento de resistencia en ensayos clínicos aleatorizados de jóvenes. Jama Pediatrics, 168 (11), 10061014.

TANAKA, H., MONAHAN, K., \& SEALS, D. 2001. Age-predicted maximal heart rate revisited. [Velocidad cardíaca máxima prevista por edad revisada]. Journal of the American College of Cardiology, 37(1), 153-156.

VARELA, A. 2014. Efectos del entrenamiento concurrente, polarizado y tradicional, sobre la condición física saludable. (Tesis Doctoral). Universidad de: Da Coruña, Coruña.

YÁNEZ, R., PULGAR, N., GUAJARDO, C., RODRÍGUEZ, F., VENEGAS, A., MACERA, E., \& ZAVALA, J. 2017. Efectos de un programa de entrenamiento polarizado: Somatotipo, composición corporal y autoestima en mujeres sedentarias. In $12^{\circ}$ Congreso Argentino de Educación Física y Ciencias 13 al 17 de noviembre 2017 Ensenada, Argentina. Educación Física: construyendo nuevos espacios. Universidad Nacional de La Plata. Facultad de Humanidades y Ciencias de la Educación. Departamento de Educación Física.

YUHASZ, M. 1977. Physical fitness manual. University of Western Ontario.

ZUSSA, D. 2017. Metabolismo de las grasas, diferentes modos de programación del ejercicio y sus efectos en la composición corporal (Doctoral dissertation, Universidad Nacional de La Plata, Argentina: Facultad de Humanidades y Ciencias de la Educación).

Cómo citar: Pirazán, J., Rivera, M., Anzola, F., \& Osuna, J. (2019). Efectos de un programa de entrenamiento concurrente sobre el perfil antropométrico y la fuerza muscular en un grupo de jóvenes universitarios. R. Actividad fis. y deporte. 6 (1): $14-31$.

Artículo de acceso abierto publicado por: Revista Digital: Actividad Física y Deporte, bajo una licencia Creative Commons CC BY-NC 4.0. 